
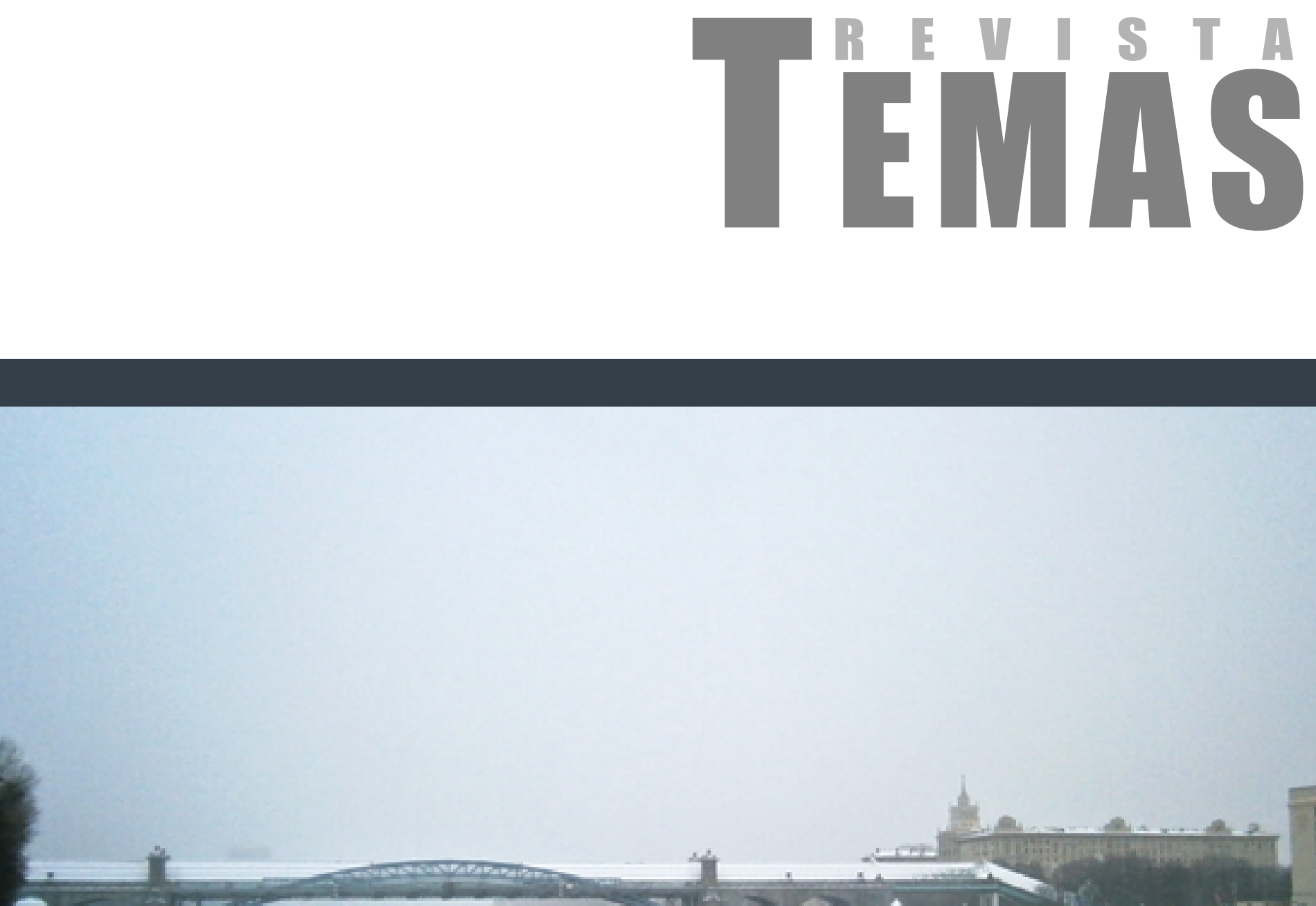
iํ $-3+2=0$
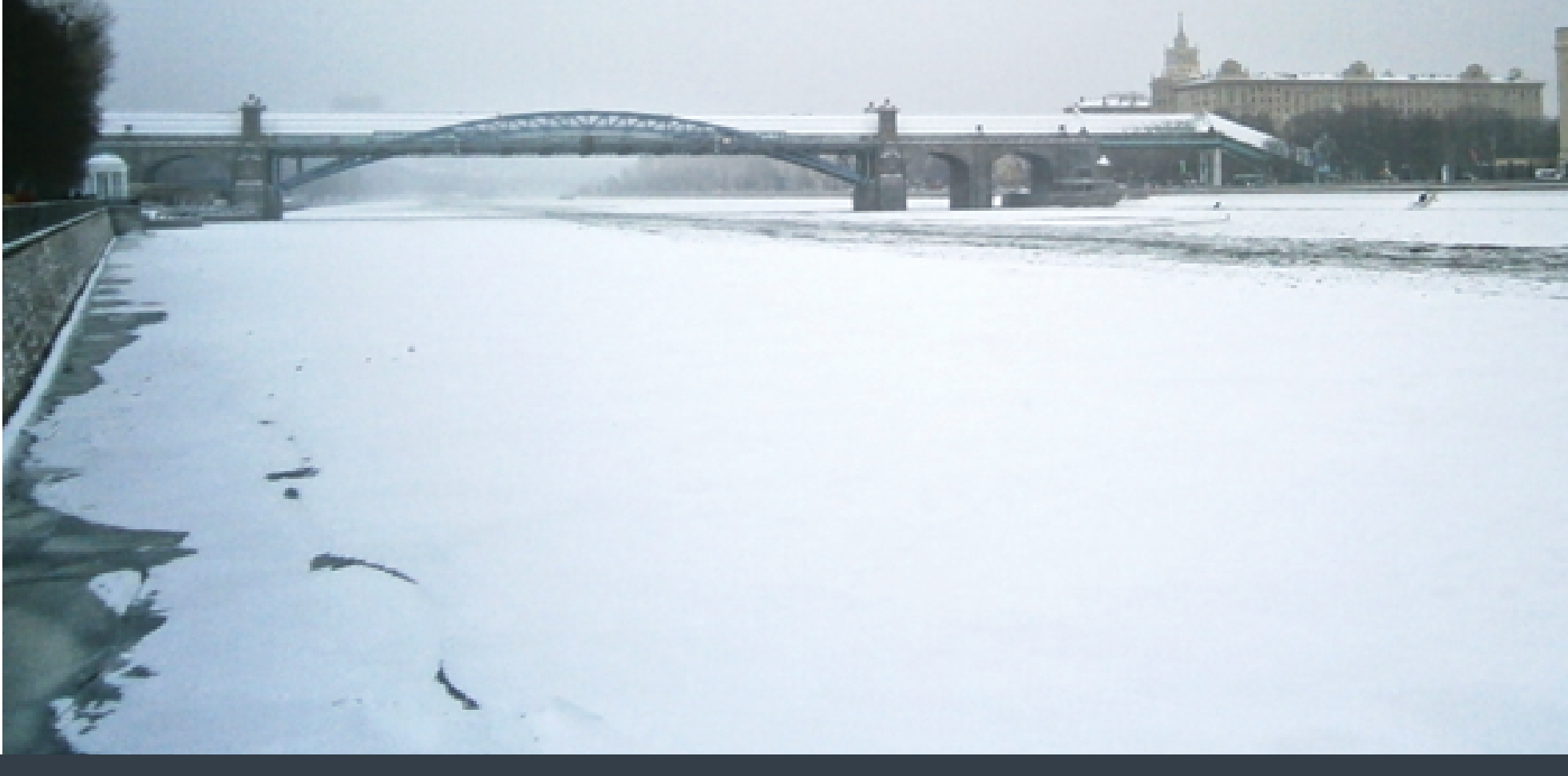

Referencia al citar este artículo:

Monroy, A.M., Suárez, P. (2018). Factores escolares asociados al aprendizaje de la física. REVISTA TEMAS, III(12), 79-96.

\title{
Factores escolares asociados al aprendizaje de la física ${ }^{1}$
}

\author{
Angélica María Monroy Córdoba ${ }^{2}$ \\ Publio Suárez Sotomonte ${ }^{3}$
}

Recibido 30 de marzo de 2017. Aceptado 1 de mayo de 2018

\section{Resumen}

Los factores escolares juegan un papel importante dentro del aprendizaje de la física. Es necesario identificarlos debido a que el docente puede con ellos mejorar el desarrollo y diseñar nuevas actividades metodológicas en el aula. En esta investigación se propone identificar, caracterizar y relacionar los factores escolares asociados con el aprendizaje de la física, además crear unas actividades metodológicas para implementar en el aula, y así lograr un mayor nivel de aprendizaje. Se tiene en cuenta como principal representante a David Ausubel, quien habla del aprendizaje significativo dentro de este carácter teórico. Se observan los tipos de aprendizajes, como: el de representaciones, de conceptos y de proposiciones.

En este artículo también se presentan fundamentados los factores escolares, que nos hablan de las características internas del estudiante, Ilamados intraescolares y los extraescolares que son los que están relacionados con directivos, docentes y autoridades municipales, aquí también se relacionan los factores motivacionales, los cuales tienen que ver directamente con el trabajo realizado en el aula.

\section{Palabras clave}

Factores, intraescolares, motivacionales, estrategia lúdica.

\section{School factors associated with the learning of physics}

\section{Abstract}

School factors play an important role in the learning of physics. This is necessary to identify because the teacher can to get better the development of your activities in the classroom and the teacher can design new methodological activities. In this research it is proposed to identify, characterize and relate the school factors associated to the learning of physics as well as to create methodological activities to implement in the classroom and thus a higher level of learning.

It is considered as main representative to David Ausubel who speaks of meaningful learning within this theoretical character we observe the types of learning among which we find the learning of representations, concepts, propositions.

This article also presents the school factors, which tell us about the internal characteristics of the student called intraschool and the extracurricular that are related to managers, teachers and municipal authorities, here also relate the motivational factors which have to See directly with the work done in the classroom.

\section{Keywords}

Intraschool factors, motivational factors, ludic strategy.

1. Artículo de investigación.

2. Magíster en Educación por la Universidad Santo Tomás. Correo electrónico: firmeangelica@yahoo.es.

3. Doctor en Informática por la Universidad Pedagógica y Tecnológica de Colombia. Correo electrónico: psuarez2002@hotmail.com 


\section{Introducción}

La educación es un derecho fundamental consagrado en nuestra Constitución Política. Al estudiar los factores escolares asociados al aprendizaje de la física en la Escuela Normal Superior Leonor Álvarez Pinzón, institución educativa de carácter público en el municipio de Tunja, es posible observar que estos factores afectan a niños, niñas y adolescentes de manera negativa o positiva. Por eso su estudio genera bases fundamentales para promover la igualdad y generar impacto dentro de las aulas estudiantiles para el manejo de los aprendizajes en física del grado décimo.

Históricamente, desde la década de los noventa, en Latinoamérica diversas entidades públicas y privadas han venido preocupándose por encontrar estos factores escolares asociados a los aprendizajes en lengua castellana y matemáticas para primaria (Miranda, 2007). La UNESCO ha impulsado también a que se estudien los factores asociados al aprendizaje, debido a que la calidad educativa de los estudiantes mejora los resultados en las pruebas nacionales e internacionales.

Estos resultados obtenidos en evaluaciones nacionales han señalado brechas en el aprendizaje entre educación pública y privada en el país, sin embargo, no es correcto afirmar que las escuelas privadas sean mejores que las públicas, sino que hay variables que se asocian al aprendizaje de la física, siendo esta un área de un alto nivel de dificultad para estudiantes en los últimos niveles de secundaria.

La asignatura de Física es de gran complejidad en los estudiantes, pero aporta al conocimiento unas características fundamentales, como: aumenta la percepción cognitiva, promueve la capacidad de pensar en forma abstracta, se encuentran analogías entre diversos fenómenos y crea el hábito de enfrentar problemas, tomar consecuentes iniciativas y establecer criterios de verdad, por último otorga confianza frente a muchas situaciones, siendo esto útil para los estudiantes del grado décimo, quienes están entre 15 y 16 años.

A esta edad el adolescente desarrolla riesgos, tales como bajo rendimiento o deserción escolar, crisis de autoridad, tiempo libre mal utilizado, segregación grupal, además con los factores asociados al aprendizaje es posible encontrar otros componentes que pueden ayudar a determinar si hay aprendizaje en la asignatura de física.

\section{Metodología}

Este capítulo tiene como propósito establecer la estrategia utilizada en la institución educativa Jorge Eliecer Gaitán del municipio de Tota, para conocer los factores asociados al aprendizaje de la física de tipo intrapersonal, también nombrados como escolares, en el grado décimo.

Las características de esta investigación son de tipo descriptivo, puesto que el propósito de la investigación es identificar los factores escolares asociados al aprendizaje de la física, y se analiza la muestra cualitativamente.

\section{Enfoque cualitativo}

Jorge Walter (2005) hace referencia en su trabajo doctoral a Dankhe (1986) para afirmar que los estudios descriptivos buscan especificar las propiedades importantes de un grupo de personas, estas propiedades en esta investigación son los factores asociados al aprendizaje de la física; y el grupo que se escogió fue el grado "Décimo C" de la Institución Educativa Jorge Eliécer Gaitán en el municipio de Tota. 
Los estudios descriptivos además buscan medir diversos aspectos, para lo cual se selecciona una serie de cuestiones y se mide cada una de ellas independientemente, para luego describir y analizar lo que se quiere investigar.

En el enfoque cualitativo, el investigador tiene actividades principales; estas pueden sintetizarse de la siguiente manera (Hernández, et al., 2003):

1. El investigador observa eventos ordinarios y actividades cotidianas tal y como suceden en sus ambientes habituales, además de cualquier acontecimiento inusual.

2. El investigador está directamente involucrado con las personas que se estudian y con sus experiencias personales.

3. El investigador adquiere un punto de vista interno (desde adentro del fenómeno), aunque mantiene la perspectiva analítica y la distancia como observador externo.

4. Utiliza diversas técnicas de investigación y habilidades sociales de una manera flexible, de acuerdo con los requerimientos de la situación.

5. Produce datos en forma de notas extensas, diagramas, mapas o cuadros humanos para generar descripciones bastante detalladas.

6. Sigue una perspectiva individual y holística (los fenómenos se conciben como un todo y no como partes).

7. Entiende a los miembros que son estudiados y desarrolla empatía hacia ellos; no solamente registra hechos objetivos fríos.

8. Mantiene una doble perspectiva: analiza los aspectos explícitos, conscientes y manifiestos, así como aquellos implí- citos, inconscientes y subyacentes. En este sentido, la realidad subjetiva en sí misma es objeto de estudio.

9. Observa los procesos sin interrumpir, alterar o imponer un punto de vista externo, sino tal como son percibidos por los actores del sistema social.

10. Es capaz de manejar paradoja, incertidumbre, dilemas éticos y ambigüedad.

\section{Estudio de casos}

El estudio de casos es una herramienta pedagógica de carácter cualitativo que ayuda a la presentación de los resultados de la investigación. Este método se basa fundamentalmente en el uso del diálogo y de las encuestas, que permiten identificar el problema generador y arrojan los resultados buscados.

Barrios, et al. (2012) afirman que el estudio de casos se ha utilizado ampliamente en la investigación, pues con esto se logra comprender la realidad social y educativa. Se observa que el estudio de casos es un estudio detallado de las unidades sociales donde se advierten circunstancias concretas. Además se infiere que: "es un estudio intensivo y profundo de uno o varios casos...".

Otros teóricos señalan que el estudio de casos se ha utilizado en diferentes investigaciones, algunas con el objetivo de conocer el modo en que un profesor expone su conocimiento profesional práctico en la escuela primaria. También se ha señalado que el estudio de casos promueve en la investigación el relacionarse profundamente con la población estudiada (Martínez, 1988).

Para empezar, se pueden observar claramente las diferencias entre el aprendizaje y el desempeño académico, en esta investigación nos referiremos únicamente al aprendizaje, para esto encontramos las 
teorías del aprendizaje en el cual el alumno adquiere conocimientos y desarrolla habilidades para desenvolverse en la vida cotidiana.

El aprendizaje significativo por lo tanto no es la mera digitación textual al pie de la letra, sino más bien ideas asimiladas donde se ve reflejado el proceso de aprendizaje y de retención.

\section{Aprendizaje significativo}

En este apartado hablaré acerca del aprendizaje significativo útil en la adquisición del conocimiento de la física, estudiaré algunas consideraciones que se hacen respecto a esta teoría y consecuencias inmediatas en el aprendizaje significativo, siendo este un referente explicativo por excelencia de gran potencialidad y aún en la modernidad vigente.

Aun cuando la teoría del aprendizaje significativo tiene una amplia experiencia de 40 años educando a estudiantes, es importante resaltar que todavía no se saben muy bien sus aspectos fundamentales; aspectos que son necesarios para lograr aprendizajes eficaces en el contexto escolar para los grados once; también es importante observar el contexto social en el que se desarrolla el aprendizaje significativo, ya que este contexto es relevante al analizar dichos aprendizajes. Ausubel presenta dos dimensiones del aprendizaje escolar, uno es el aprendizaje por recepción-descubrimiento, que está enfocado, según García (2011), a la estrategia de instrucción que se da en la escuela y, el otro aprendizaje, repetitivo-memorístico, está relacionado con la forma de aprender del alumno, este segundo aprendizaje es el que más se adquiere en el aula de física por los estudiantes, quienes realizan a diario actividades repetitivas para entender $y$ solucionar problemas cognitivos.
Tipos de aprendizaje significativo según Ausubel

Ausubel considera tres tipos de aprendizaje: aprendizaje de representaciones, aprendizaje de conceptos y aprendizaje de proposiciones.

\section{Aprendizaje de representaciones}

Según García (2011) el aprendizaje de representaciones se observa cuando el niño aprende primero palabras que representan objetos reales pero que en primera instancia no tienen categorización aunque sí significado, una vez que se conocen los conceptos se utilizan las frases que va construyendo de este y que además contengan otros conceptos, empezando con esto desarrollar el segundo tipo de aprendizaje, el aprendizaje de conceptos.

\section{Aprendizaje de conceptos}

Para Ausubel los conceptos son propiedades de los objetos, donde no se hace referencia a la diversidad de medios, sino que es válido para cualquier contexto, se utiliza para su representación el símbolo o la señal.

Muchos autores, entre ellos Bruner, destacan la importancia de los conceptos afirmando que estos sirven para:

- Reducir la complejidad del entorno.

- Identificar los objetos que hay en el mundo.

- Reducir la necesidad de un aprendizaje constante.

- Proporcionar una dirección a la actividad instrumental.

- Ordenar y relacionar clases de hechos.

De acuerdo con esto, el conducirnos a un aprendizaje de conceptos es muy 
difícil, de hecho, más difícil que los conceptos que han elaborado las ciencias básicas útiles en el desarrollo de las sociedades contemporáneas.

Según Ausubel los niños en edad pre-escolar concretan sus conceptos a través de la experiencia, en niños un poco mayores estos conceptos se van organizando a través de la asimilación y otras acomodaciones que realizan de acuerdo con su experiencia con el transcurso del tiempo.

\section{Aprendizaje de proposiciones}

Tayupe cita a Ausubel (1976) en el aprendizaje de proposiciones y afirma que:

Este tipo de aprendizaje va más allá de la simple asimilación de lo que representan las palabras, combinadas o aisladas, puesto que exige captar el significado de las ideas expresadas en forma de proposiciones.

El aprendizaje de proposiciones implica la combinación y relación de varias palabras cada una de las cuales constituye un referente unitario, luego estas se combinan de tal forma que la idea resultante es más que la simple suma de los significados de las palabras componentes individuales, produciendo un nuevo significado que es asimilado a la estructura cognoscitiva. Es decir, que una proposición potencialmente significativa, expresada verbalmente, como una declaración que posee significado denotativo (las características evocadas al oír los conceptos) y connotativo (la carga emotiva, actitudinal e idiosincrática provocada por los conceptos) de los conceptos involucrados, interactúa con las ideas relevantes ya establecidas en la estructura cognoscitiva $y$, de esa interacción, surgen los significados de la nueva proposición. Cuando conoce el significado de los conceptos puede formar frases que contengan dos o más conceptos en donde afirme o niegue algo. Así, un concepto nuevo es asimilado al integrarlo en su estructura cognitiva con los conocimientos previos".

\section{Factores escolares}

García (2011) en su texto nos habla de factores escolares que están clasificados en dos categorías: la categoría intrapersonal, que se refieren a los factores internos del alumno y los situacionales o de categoría situacional, estos tienen que ver con las variables del medio, los docentes y las situaciones en donde se lleve a cabo el aprendizaje, es decir, su contexto.

\section{Factores intrapersonales}

Las variables referentes a la categoría intrapersonal son:

- § Variables de la estructura cognitiva: La forma en cómo estén organizados, los conocimientos previos del alumno, lo que sabe y conoce acerca de un tema influyen en la asimilación de nuevo conocimiento.

- Disposición de desarrollo: Es relacionada con el desarrollo intelectual de un alumno, la disposición depende de la edad de los alumnos, los alumnos de primaria tienen más capacidades y otras modalidades para aprender que los alumnos de preescolar.

- Capacidad intelectual: El aprendizaje de un nuevo conocimiento depende también de la inteligencia, las capacidades cognitivas, habilidades para resolución de problemas y las aptitudes escolares intelectuales de un alumno.

- Variables motivacionales y actitudinales: la actitud positiva de aprender, el impulso cognitivo, la motivación de 
mejorar en la escuela sin duda son factores que influyen en el aprendizaje.

- Variables de personalidad: la personalidad, el estilo personal de aprender, los niveles de ansiedad, las diferencias individuales de cada alumno intervienen en el proceso de aprendizaje.

En cuanto a la categoría situacional las variables del aprendizaje son las siguientes:

- La práctica: La frecuencia, el sobreaprendizaje, y la retroalimentación que se tenga del conocimiento facilita su retención.

- El orden de materiales de enseñanza: Se refiere al orden interno de los contenidos escolares, los materiales que propician a aprenderlos significativamente, tienen una secuencia y están estructurados con base en la diferenciación progresiva y reconciliación integradora.

- Factores sociales y de grupo: El ambiente que exista en el aula de clases, la interacción, competencia, cooperación, estatus social etc. son variables que inciden en el aprendizaje de las materias escolares, las actitudes y valores.
- Características del profesor: Las capacidades cognitivas del profesor influyen a la hora de enseñar, así como los conocimientos en torno a la materia que imparte, su personalidad, la conducta que tenga en aula de clases y la interacción con los alumnos.

\section{Factores motivacionales y actitudinales}

Los factores que corresponden a este nivel son:

- Actitud para aprender

- Impulso cognitivo

- La motivación de mejorar en la escuela

\section{Resultados}

En las siguientes tres tablas se encuentran condensados los resultados obtenidos en las encuestas. Los nombres de los estudiantes fueron omitidos debido a que, por norma, se debe preservar su identidad.

Por esta razón se etiquetó a cada estudiante con un número de 1 a 12, siendo doce estudiantes en total. Cada factor fue escogido según los antecedentes presentados en el marco teórico de la presente investigación. 
Tabla 1. Resultados de la encuesta teniendo en cuenta los factores escolares: edad, sexo, horas de estudio y actividades

\begin{tabular}{cc|c|c|c} 
Estudiante & Edad & Género & $\begin{array}{c}\text { Horas de } \\
\text { estudio }\end{array}$ & Actividades \\
\hline 1 & 16 & FEMENINO & B & B \\
2 & 15 & FEMENINO & D & B \\
3 & 16 & FEMENINO & D & C \\
4 & 18 & FEMENINO & B & B \\
5 & 16 & FEMENINO & B & C \\
7 & 15 & FEMENINO & B & B \\
8 & 17 & FEMENINO & C & B \\
9 & 16 & FEMENINO & D & B \\
10 & 18 & FEMENINO & D & C \\
11 & 16 & FEMENINO & B & B \\
17 & 15 & FEMENINO & B & B \\
\hline 10 & 16 & FEMENINO & A & B \\
\hline
\end{tabular}

Tabla 2. Resultados de la encuesta teniendo en cuenta los factores escolares de estudios realizados antes de los 6 años, lugar de residencia, libros en casa, años reprobados

\begin{tabular}{c|c|c|c|c} 
Estudiante & $\begin{array}{c}\text { Antes 6 } \\
\text { años }\end{array}$ & $\begin{array}{c}\text { Lugar de } \\
\text { residencia }\end{array}$ & $\begin{array}{c}\text { Libros en } \\
\text { casa }\end{array}$ & Años reprobados \\
\hline 2 & Sí & B & B & 0 \\
3 & Sí & B & C & 0 \\
4 & Sí & B & A & 0 \\
5 & Sí & B & A & 0 \\
6 & Sí & B & A & 0 \\
7 & Sí & B & C & 0 \\
8 & Sí & B & A & 1 \\
9 & Sí & B & A & 1 \\
10 & Sí & B & B & 2 \\
11 & Sí & A & B & 0 \\
12 & Sí & B & B & 0 \\
\hline & Sí & B & A & 2
\end{tabular}




\begin{tabular}{ccccc|c} 
Estudiante & Computador & Estrato & $\begin{array}{c}\text { Agrado por } \\
\text { la Física }\end{array}$ & $\begin{array}{c}\text { Resultados } \\
\text { PEA }\end{array}$ \\
\hline 1 & NO & 1 & B & 2.8 \\
2 & sí & 1 & B & 4.5 \\
3 & Sí & 1 & C & 5.0 \\
4 & sí & 1 & C & 2.5 \\
5 & NO & 1 & A & 3.8 \\
6 & NO & 1 & A & 3.6 \\
7 & NO & 1 & A & 3.4 \\
8 & NO & 1 & B & 3.2 \\
9 & Sí & 1 & C & 3.5 \\
10 & Sí & 2 & C & 3.8 \\
11 & Sí & 1 & C & 2.8 \\
12 & Sí & 1 & A & 2.5
\end{tabular}

En las encuestas estructuradas los

Tabla 4. Comparación del factor EDAD con los RESULTADOS PEA resultados que se encontraron fueron los siguientes:

En el Factor escolar asociado de edad, se puede observar que esta oscila entre 15 y 18 años, razón por la cual se clasificó el grupo de adolescentes en la edad de desarrollo psicológico. Este factor se tuvo en cuenta ya que Peña (2011) lo relaciona en su investigación, tal como aparece en el marco teórico de esta investigación, y declara que su importancia radica en que este factor tiene gran influencia en el aprendizaje cuando el estudiante es adolescente. La relación que se encontró entre este factor y el aprendizaje en esta investigación lo podemos visualizar en la siguiente tabla.

\begin{tabular}{cc|}
\hline \multicolumn{2}{|c|}{ PEA } \\
\hline Edad & Resultados PEA \\
\hline 16 & 2.8 \\
15 & 4.5 \\
16 & 5.0 \\
18 & 2.5 \\
16 & 3.8 \\
16 & 3.6 \\
15 & 3.4 \\
17 & 3.2 \\
16 & 3.5 \\
18 & 3.8 \\
16 & 2.8 \\
15 & 2.5 \\
\hline 17 &
\end{tabular}


Si analizamos con porcentajes la situación de aprobación, podemos observar que los adolescentes de 15 años aprueban con un $66 \%$, los adolescentes de 16 años aprueban con el $80 \%$, y los adolescentes de 17 y 18 años aprueban con un porcentaje del $50 \%$ en ambos casos.

Tabla 5. Comparación del factor HORAS DE ESTUDIO con los RESULTADOS PEA

\begin{tabular}{|c|c|}
\hline $\begin{array}{c}\text { Horas de } \\
\text { estudio }\end{array}$ & Resultados PEA \\
\hline B & 3.2 \\
\hline D & 4.5 \\
\hline D & 5.0 \\
\hline B & 2.5 \\
\hline B & 3.8 \\
\hline B & 3.6 \\
\hline C & 3.4 \\
D & 3.2 \\
D & 3.5 \\
B & 3.8 \\
\hline B & 2.8 \\
\hline A & 2.5 \\
\hline
\end{tabular}

Se observó que seis estudiantes dedican a su estudio 1 hora semanal; estos estudiantes tienen un porcentaje de aprobación de $66 \%$. Cuatro adolescentes dedican más de dos horas semanales; su porcentaje de aprobación es de 100\%. Un adolescente no dedica ninguna hora adicional a su estudio; su porcentaje de aprobación es de $0 \%$, y un adolescente dedica dos horas semanales, con un porcentaje de aprobación de 0\%. Este factor es muy influyente en el resultado de los promedios, pues aquellos que le dedicaron una o dos horas semanales al estudio de la Física en la casa aprobaron la prueba donde se analizan aprendizajes; solo para dos estudiantes este factor no fue influyente en su aprendizaje.

En el cuarto factor se preguntó sobre la forma de realizar las actividades propuestas. La tabla comparativa con los resultados en el aprendizaje fue la siguiente:

Tabla 6. Comparación del factor ACTIVIDADES con los RESULTADOS PEA

\section{Actividades Resultados PEA}

B

B

C

B

C

B

B

B

C

B

B

B

\section{2}

5.0

3.8

2.7

3.5

2.8

2.5
El porcentaje cuantitativo de aprobación para la pregunta B fue del $55 \%$ y de la Pregunta C fue del $100 \%$.

De acuerdo con la siguiente tabla se pueden comparar los resultados de este factor con los resultados de la evaluación obtenida. 
Tabla 7. Comparación del factor AGRADO A LA FÍSICA con los RESULTADOS PEA

\begin{tabular}{c|c}
$\begin{array}{c}\text { Agrado por la } \\
\text { Física }\end{array}$ & Resultados PEA \\
B & 2.8 \\
B & 4.5 \\
C & 5.0 \\
C & 2.5 \\
A & 3.8 \\
A & 3.6 \\
A & 3.4 \\
B & 3.2 \\
C & 3.5 \\
C & 3.8 \\
C & 2.8 \\
A & 2.5
\end{tabular}

En el numeral a, cuya respuesta es que sí le agrada la Física porque es fácil de aprender, tres estudiantes aprobaron la prueba; cuantitativamente el porcentaje de aprobación para este factor es de $100 \%$. Se puede concluir que este factor es muy importante cuando la asignatura les agrada porque es fácil de aprender.

El numeral B no es representativo en esta investigación, ya que el porcentaje de aprobación es menor que el anterior, a saber: de $66 \%$; dos estudiantes aprobaron la prueba.

El numeral $C$, con un porcentaje de $60 \%$, no fue relevante en esta investigación, aun cuando se tuvieron en este numeral los mejores resultados. Por lo tanto para que existan aprendizajes positivos en el adolescente, es necesario que la asignatura sea fácil de aprender y además divertida.
En el factor escolar de estudios realizados antes de los seis años las respuestas que el adolescente podía dar según las encuestas fueron:

Sí o No.

En la tabla 8 se muestra esta relación:

Tabla 8. Comparación del factor escolar ESTUDIOS ANTES DE LOS SEIS AÑOS con los RESULTADOS PEA

\begin{tabular}{|c|c|}
$\begin{array}{c}\text { Estudios antes } 6 \\
\text { años }\end{array}$ & Resultados PEA \\
\hline sí & 2.8 \\
sí & 4.5 \\
sí & 5.0 \\
sí & 2.5 \\
sí & 3.8 \\
sí & 3.6 \\
sí & 3.4 \\
sí & 3.2 \\
sí & 3.5 \\
sí & 3.8 \\
sí & 2.8 \\
sí & 2.5
\end{tabular}

En este factor no se pudo establecer ninguna relación con el aprendizaje de la Física, pues todos los estudiantes han realizado estudios antes de los seis años. Esto se debe a que en Colombia, desde el Estado, se han venido implementando políticas educativas que favorecen a los niveles más vulnerables, y estos adolescentes fueron beneficiados con algunas de estas políticas, por lo que este factor ya no es relevante.

Al realizar la investigación se observó que las encuestas mostraron una clara 
relación entre el aprendizaje de la Física y la repitencia en la escuela. Este factor tiene una gran influencia debido a que se observa en las evaluaciones de aprendizaje que algunos adolescentes adquieren una mayor responsabilidad al momento de repetir un año; los resultados de la evaluación de aprendizajes determinaron que a estos estudiantes se les facilitó la evaluación, debido a que tenían un conocimiento más seguro en Matemáticas, por lo tanto, desarrollaron con mayor facilidad los problemas que se les plantearon en la presente investigación.

Al realizar la pregunta abierta: ¿Qué expectativas tiene con respecto a la asignatura de Física? Los adolescentes entienden que la Física les puede servir para cuando estén desempeñando alguna labor, dependiendo del caso. También que esta es fundamental para la vida, dependiendo de la profesión que se escoja. $A$ un adolescente de 17 años le gusta la asignatura porque en un futuro le ayudará a la vida diaria.

Angie, de 15 años, dijo: "la asignatura de Física es buena para mi vida porque así nos puede ayudar a conseguir un buen trabajo, y puedo cumplir sueños y metas propuestas".

El factor Vivienda rural o Vivienda urbana también está relacionado directamente con el marco teórico explicado por Treviño (2010), quien afirma que este factor es relevante en su investigación y que se adquieren buenos resultados cuando los adolescentes pertenecen a la zona urbana.

La tabla 9 nos muestra los resultados comparativos de este factor con la prueba de evaluación de aprendizajes.

Tabla 9. Comparación del factor escolar LUGAR DE RESIDENCIA con Ios RESULTADOS PEA

Lugar de residencia

B

B

B

B

B

B

B

B

B

A

B

B

\section{Resultados PEA}

4.5

5.0

2.5

3.8

3.6

3.4

3.2

3.5

3.8

2.8

2.5

Se puede observar que solo una adolescente del total de la muestra vive en el casco urbano del municipio y aprobó la prueba de aprendizajes; por lo tanto se recomienda tener en cuenta este factor para futuros estudios comparativos $y$ poder así observar la incidencia de este factor, pues en esta investigación no fue posible analizarlo ampliamente, debido a que la mayoría de la población estudiada en esta investigación fue rural.

Para el factor escolar relacionado con los libros complementarios de estudio en la casa. Los resultados de esta pregunta y que se tuvieron en cuenta en esta investigación fueron los presentados en la tabla 10: 
Tabla 10. Comparación del factor escolar LIBROS EN CASA con Ios RESULTADOS PEA

\begin{tabular}{c|c} 
Libros en casa & Resultados PEA \\
B & 2.8 \\
C & 4.5 \\
A & 5.0 \\
A & 2.5 \\
A & 3.8 \\
C & 3.6 \\
A & 3.4 \\
A & 3.2 \\
B & 3.5 \\
B & 3.8 \\
B & 2.8 \\
A & 2.5
\end{tabular}

Aquí se puede ver que los adolescentes que contestaron la pregunta $C$ aprobaron la prueba de aprendizajes asociada a esta investigación; mientras que los que contestaron las preguntas $A$ y $B$ reprobaron y aprobaron la prueba; lo que indica que no puede establecerse una relación entre su respuesta y el resultado de la prueba. Para poder aprender Física con resultados positivos es necesario adquirir libros y consultarlos adecuadamente, para así apropiarse del aprendizaje y obtener buenos resultados.

Los porcentajes de aprobación de este factor son los siguientes: para quienes respondieron $A$ (de 1 a 5 libros) el porcentaje de aprobación es de $66 \%$; para los que respondieron $B$, es de $50 \%$, y para los que respondieron $\mathrm{C}$ el porcentaje de aprobación es de $100 \%$. Así se concluye que los estudiantes que adquieren más libros en sus hogares tienen más facilidad para aprobar la asignatura, debido a que tienen donde consultar sus tareas y actividades complementarias. El porcentaje de $66 \%$ se debe a que, cuando se habló individualmente con los estudiantes, se dijo que ellos deben tratar de estudiar con lo poco que los padres pueden facilitarles; sin embargo, se recomienda tener políticas institucionales para mejorar este aspecto, ya que es de gran importancia en el aprendizaje, especialmente de la Física.

Al analizar el factor escolar Años reprobados podemos observar que este factor puede ser determinante, dependiendo de las otras condiciones asociadas a esta investigación. Independientemente, este resultado no aportó información relevante; pero al observar el adolescente en forma personalizada, se pudo concretar que la prueba diagnóstica es aprobada más fácilmente cuando el adolescente no ha reprobado ningún grado durante su estadía escolar.

Tabla 11. Comparación del factor escolar asociado AÑOS REPROBADOS con RESULTADOS PEA

Años reprobados

Resultados PEA

0

2.8

0

4.5

0

5.0

0

2.5

0

3.8

0

3.6

1

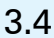

1

3.2

2

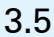

0

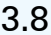

0 
Al analizar los porcentajes en este factor encontramos que los estudiantes que no reprueban ningún año tienen un porcentaje de aprobación del $62 \%$; los adolescentes que reprobaron un grado aprobaron los resultados de las pruebas de aprendizaje en un $100 \%$ y para los estudiantes que reprobaron dos grados, el porcentaje de aprobación fue del $50 \%$.

Los adolescentes evaluados durante la investigación presentaron sus sugerencias indicando que el factor escolar de Adquisición de libros y computadoras es primordial para la realización de sus actividades académicas, y que no solo les sirve para la asignatura de Física, sino para complementar sus estudios en todas las asignaturas.

Este factor está relacionado con la pregunta número 10 y número 11 en la que se pregunta además por los materiales utilizados, como computadores en la casa y en la institución, indispensables, igualmente en el aprendizaje de los estudiantes.

Se pudo visualizar un buen desempeño de la prueba en una adolescente que no cumplía con ninguno de los factores relacionados anteriormente. Se dialogó con ella de forma muy discreta, ya que no quería figurar en la investigación, por lo que le propuse que no saldría su nombre. Me pareció interesante el resultado satisfactorio obtenido en la prueba, a pesar de que, en el resultado de la encuesta estructurada, se observó que no había estudiado antes de los 6 años y que toda la primaria la hizo en una escuela rural. Sin embargo, ella le dedica entre una y dos horas de estudio diarias a todas las asignaturas y, según el diálogo que tuvimos, le encantó que yo fuera su docente de Física, pues dijo que yo le explicaba lo que ella no había logrado entender en casa y que le habían gustado todas las formas en que le dictaba la asignatura, pero que, de todas formas, prefería la explicación formal en clase.

Además, con respecto al tema evaluado, afirmó que ese tema ya se lo había explicado una profesora de ciencias en el colegio, y que con la explicación que yo le di, le quedó completamente claro. No tuvo ningún error en su evaluación de desempeño, por lo que, además de observar el factor asociado, se pudo comprobar el aprendizaje satisfactorio en esta adolescente.

La impresión que causó esta investigación en los adolescentes de este municipio de Tunja en la Escuela Normal Superior Leonor Álvarez Pinzón, en el grado décimo, por ejemplo en Angie Paola fue positiva y agradable, pues, además de verse motivada con la asignatura, se le incentivó a que colaborara con las encuestas para que al final del año tuviera una recompensa adicional, motivada por la calificación definitiva en la asignatura.

\section{Elaboración de la estrategia metodológica para enseñanza de aprendizajes}

La enseñanza del tema propuesto en esta investigación se diseñó de acuerdo con el siguiente esquema. Se trata de una estrategia metodológica de enseñanza tradicional que se ha venido desarrollando, así: 
Figura 2. Triangulación de la metodología aplicada para desarrollar el tema de densidad

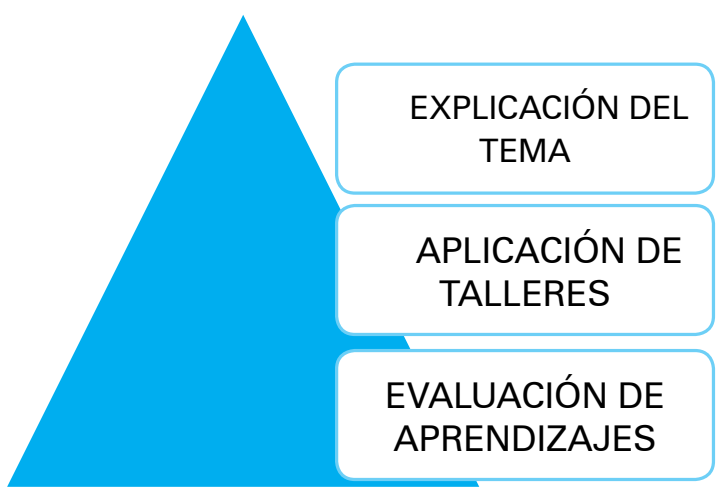

Este esquema se realizó así debido a que las estudiantes vienen desarrollando esta metodología en las diferentes asignaturas. Por eso los talleres se diseñaron de acuerdo con el avance que estas alumnas han tenido en la asignatura de Física y con su nivel de pensamiento.

Para proponer mi estrategia metodológica, me basé en el hecho de que al estudiante se le facilita la enseñanza cuando se atreve a pensar jugando y resuelve así los diferentes problemas en el marco de una actividad lúdica común. Escogí el juego de parqués, pues es un juego que resulta compatible con el aprendizaje de la Densidad para la resolución de problemas físicos adecuados para la enseñanza en el grado Décimo.

\section{Evaluar jugando}

La evaluación consiste en realizar por parejas un torneo de parqués, en donde hay 40 problemas de Densidad y Volumen, que se hará de la siguiente manera:

\section{Instrucciones de la evaluación}

Las estudiantes se dividirán en tres grupos de cuatro jugadores. En cada lanzamiento se realizará una pregunta que, si se responde correctamente, permite avanzar, si no, el jugador debe permanecer en su lugar y sigue el otro jugador.

\section{Para el evaluador:}

Por ejemplo, si el estudiante respondió 40 problemas, se divide entre el número de problemas que se le plantearon y se multiplica por la mayor calificación posible, que para esta investigación es de 5.0. El estudiante ganador del juego obtiene la mayor calificación.

\section{Normas del juego}

La salida es con pares; si saca par de 6 o par de 1, saca todas las fichas.

Si llega al seguro, no tiene que responder ningún problema.

El estudiante que no coma a su compañero, regresa con la ficha al inicio.

Al sacar tres pares seguidos, corona una de las fichas, así esta se encuentre en la salida.

Los problemas tienen que estar bien desarrollados para poder avanzar; no olvidar la notación científica en todos los problemas y las unidades. lumen

Treinta problemas de Densidad y Vo-

1. Calcular el volumen de un cubo de lado $\mathrm{I}=8 \mathrm{~cm}$

2. Calcular el volumen de un cubo de lado I $=34 \mathrm{~cm}$

3. Calcular el volumen de un cubo de lado I $=23 \mathrm{~cm}$

4. Calcular el volumen de un cubo de lado $\mathrm{I}=6 \mathrm{~cm}$

5. Calcular el volumen de un cubo de lado $\mathrm{I}=7,5 \mathrm{~cm}$

6. Calcular el volumen de un prisma de lado $\mathrm{I}=4,2 \mathrm{~cm}$, ap $=4 \mathrm{~cm}$ y altura $\mathrm{h}$ $=2,4 \mathrm{~cm}$ 
7. Calcular el volumen de un prisma de lado I $=3,5 \mathrm{~cm}$, ap $=4,5 \mathrm{~cm}$ y altura $\mathrm{h}=3,2 \mathrm{~cm}$

8. Calcular el volumen de un prisma de lado I $=5 \mathrm{~cm}$, ap $=2,4 \mathrm{~cm}$ y altura $\mathrm{h}$ $=1,2 \mathrm{~cm}$

9. Calcular el volumen de un prisma de lado I $=12,3 \mathrm{~cm}$, ap $=2,3 \mathrm{~cm}$ y altura $\mathrm{h}=24,3 \mathrm{~cm}$

10. Calcular el volumen de un prisma de lado I $=6 \mathrm{~cm}$, ap $=4,9 \mathrm{~cm}$ y altura $\mathrm{h}=20,3 \mathrm{~cm}$

11. Calcular el volumen de un cilindro de radio $\mathrm{r}=2 \mathrm{~cm}$ y altura $\mathrm{h}=3 \mathrm{~cm}$

12. Calcular el volumen de un cilindro de radio $r=2,6 \mathrm{~cm}$ y altura $h=3,2 \mathrm{~cm}$

13. Calcular el volumen de un cilindro de radio $r=3,4 \mathrm{~cm}$ y altura $h=4,2 \mathrm{~cm}$

14. Calcular el volumen de un cilindro de radio $r=23,6 \mathrm{~cm}$ y altura $\mathrm{h}=32,3 \mathrm{~cm}$

15. Calcular el volumen de un cilindro de radio $r=24,5 \mathrm{~cm}$ y altura $h=43,2 \mathrm{~cm}$

16. Calcular el volumen de una esfera de radio $r=12,4 \mathrm{~cm}$

17. Calcular el volumen de una esfera de radio $r=3,5 \mathrm{~cm}$

18. Calcular el volumen de una esfera de radio $r=53 \mathrm{~cm}$

19. Calcular el volumen de una esfera de radio $r=24 \mathrm{~cm}$

20. Calcular el volumen de una esfera de radio $r=2 \mathrm{~cm}$

21. Calcular la densidad de un cilindro de radio $r=26,7 \mathrm{~cm}$ y altura $h=3 \mathrm{~cm}$

22. Calcular la densidad de un cilindro de radio $r=21,4 \mathrm{~cm}$ y altura $h=3,5 \mathrm{~cm}$
23. Calcular la densidad de un cilindro de radio $r=22,4 \mathrm{~cm}$ y altura $h=32 \mathrm{~cm}$

24. Calcular la densidad de un cilindro de radio $r=21,8 \mathrm{~cm}$ y altura $h=12,4 \mathrm{~cm}$

25. Calcular la densidad de un cilindro de radio $r=2 \mathrm{~cm}$ y altura $h=304 \mathrm{~cm}$

26. Calcular la densidad de una esfera de radio $r=203 \mathrm{~cm}$

27. Calcular la densidad de una esfera de radio $r=212 \mathrm{~cm}$

28. Calcular la densidad de una esfera de radio $r=28 \mathrm{~cm}$

29. Calcular la densidad de una esfera de radio $r=23 \mathrm{~cm}$

30. Calcular la densidad de una esfera de radio $r=2,7 \mathrm{~cm}$

\section{Conclusiones}

Los Factores Escolares Asociados al aprendizaje de la Física ponen en evidencia los problemas de aprendizaje de los estudiantes y deben ser evaluados por el docente con el fin de cambiar las estrategias metodológicas para que sean didácticas y en contexto con la institución.

La implementación de la investigación permitió a las estudiantes desarrollar habilidades para la comprensión del concepto de Densidad, por medio de la resolución de talleres didácticos.

El trabajo por equipos de aprendizaje “Metodologías centradas en equipos de aprendizaje (MICEA)", fue una estrategia clave en el desarrollo de la investigación, porque permitió afianzar conocimientos, fortalecer procesos en los estudiantes y utilizar nuevos métodos de enseñanza dentro del aula.

Los Factores Escolares más influyentes en el marco de la investigación fueron los 
Años reprobados, la Cantidad de libros en casa y la Cantidad de horas utilizadas para el desarrollo de las tareas y contenidos del tema propuesto.

Dentro de los procesos de enseñanza-aprendizaje se debe permitir que el estudiante comparta su conocimiento con otros estudiantes, debido a que la socialización permite el aprendizaje de normas para convivir y el fortalecimiento de los lazos afectivos. En el aula se deben favorecer espacios de resolución de problemas relacionados con la Física, mediante la implementación de métodos y técnicas innovadoras y lúdicas, que son elementos importantes en el desarrollo formativo, académico y profesional del estudiante.

El contexto sociocultural de los estudiantes influye directamente en el rendimiento académico de los estudiantes y debe relacionarse con las estrategias de enseñanza utilizadas, que para contextos como el nuestro pueden ser lúdicas y para contextos más amplios deben ser mejor estructuradas, para lograr aprendizajes efectivos a largo plazo y con mayor profundidad en el conocimiento explicado.

Una vez identificados los factores escolares más influyentes en la asignatura de Física en el grado décimo de la Escuela Normal Superior Leonor Álvarez Pinzón, se propone desde la escuela abordar estrategias lúdico-pedagógicas que involucren a las adolescentes en el proceso de aprendizaje, siendo ellas las generadoras como nuevas docentes de las estrategias metodológicas para el aprendizaje de la física dentro del margen de las competencias gubernamentales; utilizando la creatividad e innovación y analizando la edad para la cual se realizarán cada uno de estas estrategias metodológicas; es decir, no solo utilizar el tema de densidad sino diseñar paso a paso el proceso de cada clase para llevarlo a la práctica, y que sea en cursos más avanzados donde se fundamente pedagógicamente cada estrategia.

\section{Referencias}

Amaro, R. (2008). Diagnóstico de los factores asociados a la práctica pedagógica desde la perspectiva del docente y los estudiantes. Universidad Central de Venezuela, Caracas, Venezuela.

Araiza, O. (2012). Temas selectos de física para bachillerato. Densidad y peso específico. Recuperado de http://tslfbachillerato.blogspot. com.co/2012/12/densidad-y-peso-especificodensidad-en.html

Arias, M. (s.f.). Física: Guía práctica para bachillerato. Recuperado de http://matebrunca.com/wpcontent/uploads/2014/06/fisica-Fluidos.pdf

Backhoff, E., Bouzas, A., Contreras, C., Hernández, E., y García, M. (2007). Factores escolares y aprendizaje en México. El caso de la educación básica. Instituto Nacional para la Evaluación de la Educación. México D.F, México.

Barrios, I., González, J., Padín, L., Peral, P., Sánchez, I., Y Tarín, E. (2012). El estudio de casos. Universidad Autónoma de Madrid, España.

Baquero, M. (2012). Instructivo para la presentación de trabajos. (pp. 2, 3 y 6). Licenciatura en Pedagogía Infantil. Universidad de la Sabana, Chía.

Betancourt, R., Guevara, L., y Fuentes, E. (2011). El taller como estrategia didáctica, sus fases componentes para el desarrollo de un proceso de cualificación en el uso de tecnologías de la información y la comunicación (TIC) con docentes de lenguas extranjeras. Caracterización y retos. Universidad de la Salle. Bogotá, Colombia.

Blanco, E. (2008). Factores escolares asociados a los aprendizajes en la educación primaria mexicana: un análisis multinivel. Revista electrónica Iberoamericana sobre calidad, eficacia y cambio en educación, 6(1).

Blanco, R. (2008). Eficacia escolar y factores asociados en América Latina y el Caribe. Santiago, Chile.

Cano, A. (2005). Las técnicas de grupo, las reuniones de trabajo. Animación social y de grupo. Curso. Recuperado de http://www2.ulpgc.es/hege/ almacen/download/38/38207/tema 5 tecnicas_de

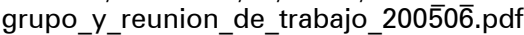

Caso, J. (2010). Factores personales, escolares y familiares que afectan el rendimiento académico en secundaria. Unidad de Evaluación Educativa. Baja California, México.

Centro de Escritura Javeriano. (s.f.). Normas APA. Recuperado de http://portales.puj.edu.co/ ftpcentroescritura/Recursos/Normasapa.pdf

Cervini, R. (2003). Factores del logro de aprendizaje de los alumnos en matemática y lengua del tercer y cuarto grado de la educación básica en 13 estados de México. Puebla, México.

Chehaybar, E., y Kuri. (2012). Técnicas para el aprendizaje grupal. Grupos numerosos. Universidad Autónoma de México, México.

Chumba, R. (2009). El aprendizaje cooperativo y la deserción escolar en licenciatura en contaduría y administración del Centro de Estudios Superiores. (Tesis para grado de Maestra en Innovación Educativa). Mérida de Yucatán, México. 
Cid, P., Díaz, A., Pérez, M., Torruella, M., y Valderrama, M. (2008). Agresión y violencia en la escuela como factor de riesgo del aprendizaje escolar. Ciencia y Enfermería, XIV(2), 21-30. Universidad de Concepción, Chile.

Cobo, E. (2008). Una propuesta para el aprendizaje significativo de los estudiantes de la escuela San José de La Salle de la ciudad de Guayaquil. Universidad Andina Simón Bolívar. Guayaquil, Ecuador.

Coll, C. (1985). Acción, interacción y construcción del conocimiento en situaciones educativas. Universidad de Barcelona. Barcelona, España.

Cornejo, R., y Redondo, J. (2007). Variables y factores asociados al aprendizaje escolar. Una discusión desde la investigación actual. Estudios Pedagogía, 33(2). Valdivia.

Díaz, F. (2003). Cognición situada y estrategias para el aprendizaje significativo. Universidad Nacional Autónoma de México, México.

Díaz, F., y Hernández, G. (2010). Estrategias docentes para un aprendizaje significativo. Una interpretación constructivista. (2a . ed.). Mc Graw-Hill. México.

Douglas, C., Bernaza, G., y Corral, R. (2006). Una propuesta didáctica para el aprendizaje de la física. Revista Ibero-Americana de Educación, 5(37).

Enríquez, C. (2008). Factores de riesgo asociados a bajo rendimiento académico en escolares de dos instituciones públicas de Bogotá. Universidad de CEES. Medellín, Colombia.

Fernández, L. (2006). ¿Cómo analizar datos cualitativos? Universitat de Barcelona, Barcelona. España.

Flórez, J.A., y. Barrientos, S. (2008, abril). Identificación de los factores asociados al logro académico de los alumnos de primaria y secundaria en Sonora, (155), 53-57.

Flórez, J.A., y Barrientos, S. (2010). Factores escolares y extraescolares asociados al logro académico de los alumnos de educación primaria y secundaria en español y matemáticas en Sonora. Instituto de Innovación y Evaluación Educativa del Estado de Sonora. Hermosillo, Sonora México.

Gámez, M., y Torres, C. (2012). Las técnicas de grupo como estrategia metodológica en la adquisición de la competencia de trabajo en equipo de alumnos universitarios. Journal for educators Teachers an Trainers, 4, 14-25.

García, D. (2011). El concepto de aprendizaje significativo de David Ausubel Joseph Novak. La construcción del concepto mediante un modelo de conocimiento. (Tesis para grado de Licenciada en Ciencias de la Educación). Cuernavaca, México.

Gilda, D., Girelli, M., y Reynoso, M. (2011). Aprendizaje activo de la física en alumnos de nivel secundario: Pre test de laboratorio sobre transformación de energía eléctrica en térmica. Universidad de La Pampa. La Pampa, Argentina.

Gutiérrez, E. (1988). Trabajo social y la deserción escolar escuela secundaria técnica No8 Minatitlan. (Tesis presentada a la Facultad de Trabajo Social de la Universidad Veracruzana. México.

Hein, A. (2004). Estudio exploratorio descriptivo de caracterización de factores de riesgo asociados al abandono escolar y sus encadenamientos, en jóvenes de escasos recursos de 12 y 20 años, pertenecientes a tres centros urbanos. Universidad de Chile. Chile.

Heredia, Y. (2007). Factores que afectan el desempeño académico de los alumnos de escuelas primarias públicas en Nuevo León. Memorias del IX Congreso Nacional de Investigación Educativa. Mérida, México.
Hernández, S. Et al. (2006). Metodología de la Investigación. Mac Graw Hill.

Herrera, D., y Aguilar, J. (s.f.). Juego didáctico para construir volúmenes geométricos deformables. Universidad de Chile. Chile.

López, O. (s.f.). Manual de estilo APA. (6 $6^{\mathrm{a}}$ ed.). Recuperado de http://es.slideshare.net/gonzaramirezg/apacitacin-estilo-fcil-gua-normas-sexta-edicinestiloapa-sextaedicin

Martínez, M. (1988). La investigación cualitativa. Revista de Investigación en Pedagogía, 9(1), 125-128.

Miranda, L. (2007). Factores asociados al rendimiento escolar y sus implicancias para la política educativa del Perú. Recuperado de http://umc.minedu.gob. pe/factores-asociados-al-rendimiento-escolar-ysus-implicancias-para-la-politica-educativa-delperu/

Moreno, F., Marthe, N., y Rebolledo, L. (2010). Cómo escribir textos con normas internacionales APA IEEE, MLA, Vancouver e Icontec. Barranquilla: Ediciones Úninorte.

Navarro, R. (s.f.). Factores asociados al rendimiento académico. Revista Iberoamericana de Educación. Universidad Cristóbal Colón. México.

Ojeda, A., Díaz, F., González, L., Pineda, P., y Hernández, M. (2007). Los mapas conceptuales: una poderosa herramienta para el aprendizaje significativo. ACIMED. Recuperado de http://bvs.sld.cu/revistas/ aci/vol15 05 07/aci09507.htm.

Peña, E. (2011). Modelos multinivel de los factores de eficacia escolar en el programa PISA. España: Universidad de Oviedo.

Pérez, A. (2007). Factores asociados con el bajo rendimiento académico en alumnos de $2^{\circ}$ año de la Escuela Secundaria Técnica No 38 "José María Morelos y Pavón". (Tesis para Licenciado en Psicología). Universidad Autónoma del Estado de Hidalgo. México.

Quintana, E. (2006). Metodología de la investigación cualitativa. Lima: UNMSM.

Ramírez, M.H. (2009). Aplicación del sistema 4MAT en la enseñanza de la física a nivel universitario. (Tesis para doctor en Física Educativa). Instituto Politécnico Nacional. México, D.F.

Regla, M. (2008). Desarrollo de estrategias de aprendizaje en los alumnos de la carrera de ingeniería en mecanización agropecuaria de la Universidad Ciego de Ávila a partir de la disciplina Física. España: Universidad de Granada.

Rey, F. (2008). Utilización de los mapas conceptuales como herramienta evaluadora del aprendizaje significativo del alumno universitario en ciencias con independencia de su conocimiento de la metodología. Barcelona: Universitat Ramón Llull.

Rico, C. (2011). Diseño y aplicación de ambiente virtual de aprendizaje en el proceso de enseñanzaaprendizaje de la física en el grado décimo de la l. E. Alfonso López Pumarejo de la ciudad de Palmira. (Trabajo final para magíster en Enseñanza de las Ciencias Exactas y Naturales). Universidad Nacional de Colombia, Sede Palmira, Valle del Cauca, Colombia.

Rivera K. (2014). Factores asociados a la repitencia desde la percepción de alumnos y docentes. El Salvador: Universidad Don Bosco.

Román, M. (2013). Factores asociados al abandono y la deserción escolar en América Latina: Una mirada en conjunto. Revista Iberoamericana sobre Calidad, Eficacia y Cambio en Educación, 11(2).

Rodríguez, G., Gil, J., y García, E. (1996). Metodología de la investigación cualitativa (pp. 2, 10 y 15). España. 
Rodríguez, M. (2011). La teoría del aprendizaje significativo: una revisión aplicable a la escuela actual. Revista Electrónica de Investigación, 3(1).

Salazar, R., Salazar, E., Flórez, N., y Luna, M. (2009). Factores asociados al logro educativo. Un enfoque centrado en el estudiante. México: Facultad Latinoamericana de Ciencias Sociales. Flacso México.

Sánchez, M. (s.f). La evaluación en la enseñanza de la física como instrumento de aprendizaje. Valencia: Universitat de Valencia.

Sevilla, C. (1994). Los procedimientos en el aprendizaje de la física. Valencia: IES Isabel de Villena.

Suárez, N., y Villalobos, J. (2010). Tesis de grado $e$ investigación cualitativa. Mérida: Archivo Arquidiocesano de Mérida. (Serie: Estudios,11).

Tayupe, M. (1976). Teoría del aprendizaje significativo de David Ausbel

Torres, S., Gonzáles, A., y Vavilova, I. (2015). La cita y la referencia bibliográfica: Guía basada en las normas APA. Buenos Aires: Biblioteca Central UCES.
Treviño, E. (2010). Factores asociados al logro cognitivo de los estudiantes de América Latina y el Caribe. Santiago de Chile: Laboratorio Latinoamericano de Evaluación de la Calidad de la Educación.

Universo de Fórmulas. (s.f.). Volumen del prisma hexagonal regular. Recuperado de http://www. universoformulas.com/matematicas/geometria/ volumen-prisma-hexagonal/

Velázquez, C. (2013). Análisis de la implementación del aprendizaje cooperativo durante la escolarización obligatoria en el área de Educación Física. Valladolid: Universidad de Valladolid.

Velázquez, W. (2013). Estilos de aprendizaje y rendimiento académico en estudiantes de grado $9^{\circ}$ de básica secundaria. Medellín: Universidad de Antioquia.

Walter,J. (2005). Tesis Doctoral. Universidad de París.

Zabala, S. (2009). Guía a la redacción en el estilo APA, ( 6 a ed.). Biblioteca de la Universidad Metropolitana. 